\title{
WEAK $n$-HOMOGENEITY IMPLIES WEAK $(n-1)$-HOMOGENEITY
}

\author{
MORTON BROWN
}

Introduction and definitions. Let $\tilde{S}$ be a set of points. By an $n$ subset of $\widetilde{S}$ we shall mean a subset of $\tilde{S}$ consisting of precisely $n$ points. Let $S$ be a subset of $\widetilde{S}$ and $G$ a group of 1-1 transformations of $\tilde{S}$ upon itself. Then $G$ is weakly n-transitive over $S$ if for any two $n$-subsets $X_{1}, X_{2}$ of $S$ there exists a $g \in G$ such that $g\left(X_{1}\right)=X_{2}$. (It is not required that $g(S) \subset S$.) In particular if $S=\widetilde{S}, \widetilde{S}$ is a topological space, and $G$ is the group of homeomorphisms of $\widetilde{S}$ upon itself, then the space $S$ is said to be weakly $n$-homogeneous. ${ }^{1}$

The purpose of this paper is to prove that an infinite weakly $n$ homogeneous topological space is weakly $(n-1)$-homogeneous $(n>1)$.

Theorem 1. Let $\tilde{S}$ be a set and let $n, k$ be fixed positive integers $(1<n \leqq k)$. Let $S$ be a $k$-subset of $\tilde{S}$ and let $G$ be weakly $n$-transitive over $S$. Then a sufficient condition that $G$ be weakly $(n-1)$-transitive over $S$ is that g.c.d. $\left(n,\left(C_{k, n-1}\right)\right)=1$.

Proof. Let $X_{1}, X_{2}, \cdots, X_{\left(C_{k, n}\right)}$ be the $\left(C_{k, n}\right)$ different $n$-subsets of $S$. We shall impose a convenient ordering on the points of each $X_{i}$. Let $X_{1}=a_{11} \cup a_{12} \cup \cdots \cup a_{1 n}$. Let $V_{1}$ be the ordered set

$$
\left\langle a_{11}, a_{12}, \cdots, a_{1 n}\right\rangle \text {. }
$$

Now for each $X_{i}$ there is a $g_{i}$ in $G$ such that $g_{i}\left(X_{1}\right)=X_{i}$. Let $V_{i}$ be the ordered set $\left\langle g_{i}\left(a_{11}\right), g_{i}\left(a_{12}\right), \cdots, g_{i}\left(a_{1 n}\right)\right\rangle$. It will be convenient to envision the $\left(C_{k, n}\right) \times n$ matrix

$$
A=\left[\begin{array}{l}
V_{1} \\
V_{2} \\
\vdots \\
V_{\left(C_{k, n}\right)}
\end{array}\right] .
$$

The transformation $g_{i}$ may be thought of as a projection of the first

Presented to the Society, April 24, 1954 under the title $N$-homogeneity implies $N-1$ homogeneity; received by the editors October 23, 1958.

${ }^{1}$ The definitions of higher homogeneity in the literature differ. The referee has suggested the following terminology (which the author adopts in this paper): $n$-homogeneity refers to ordered $n$-tuples, weak $n$-homogeneity refers to $n$-sets. This terminology coincides with the more standard concepts of transitivity and weak transitivity of groups operating on a set. 
row of $A$ onto the $i$ th row. The transformation $g_{j} g_{i}^{-1}$ projects the $i$ th row onto the $j$ th row.

For $r=1,2, \cdots, n$ let $A_{r}$ be the $\left(C_{k, n}\right) \times(n-1)$ matrix obtained by striking out the $r$ th column of $A$. Let $\{M\}$ denote the class of different $(n-1)$-subsets of $S$. Suppose $M \in\{M\}$. For notational purposes we shall say that $M$ occurs as a row in $A_{r}$ if the elements of $M$ are the elements of some row of $A_{r}$.

1.1. Clearly, if $M_{1}, M_{2} \in\{M\}$ and $M_{1}, M_{2}$ occur respectively as the $\sigma$ th and $\tau$ th rows in the same matrix $A_{r}$, then $g_{\sigma} g_{\tau}^{-1}\left(M_{2}\right)=M_{1}$.

Let $M_{0}$ be a fixed element of $\{M\}$. We define inductively a sequence $C_{1}, C_{2}, \cdots$ of subsets of $\{M\}$ as follows:

$C_{1}=\left\{M \in\{M\} \mid\right.$ for some integer $r, M$ and $M_{0}$ occur as rows in $\left.A_{r}\right\}$,

$$
C_{i+1}=\left\{M \in\{M\} \mid \begin{array}{l}
\text { for some integer } r, M \text { and an element } \\
\text { of } C_{i} \text { occur as rows in } A_{r}
\end{array}\right\} .
$$

1.2. $C_{1} \subset C_{2} \subset \cdots$.

1.3. If $M_{1}, M_{2} \in C_{i}$ then for some $g$ in $G, g\left(M_{1}\right)=M_{2}$.

1.4. There is an integer $q$ such that $C_{q}=C_{q+i}$ for every positive integer $i$.

The proof of 1.2 follows directly from the definition of $C_{i}$, and 1.4 follows from 1.2 and the fact that $S$ is finite. We prove 1.3 by induction on $i$. Suppose $M_{1}, M_{2} \in C_{1}$. Then for some $r, M_{1}$ and $M_{2}$ occur respectively as the $\sigma$ th and $\tau$ th rows of $A_{r}$. Then $g_{\tau} g_{\sigma}^{-1}\left(M_{1}\right)=M_{2}$. Now suppose $M_{1}, M_{2} \in C_{i+1}$. Then there exist $M_{3}, M_{4} \in C_{i}$ and integers $r, s$ such that $M_{1}$ and $M_{3}$ occur as rows of $A_{r}$ and $M_{2}$ and $M_{4}$ occur as rows of $A_{s}$. Hence by 1.1 there exist $g_{1}, g_{2} \in G$ such that

$$
\begin{aligned}
& g_{1}\left(M_{1}\right)=M_{3}, \\
& g_{2}\left(M_{2}\right)=M_{4} .
\end{aligned}
$$

Since $M_{3}, M_{4} \in C_{i}$ there is a $g_{3}$ in $G$ such that

$$
g_{3}\left(M_{3}\right)=M_{4} \text {. }
$$

Hence $g_{2}^{-1} g_{3} g_{1}\left(M_{1}\right)=M_{2}$.

As a corollary we have:

1.5. If $M \in C_{q}$ and $M$ occurs as a row in $A_{r}$, then each row of $A_{r}$ contains an element of $C_{q}$.

1.6. Each $M \in\{M\}$ occurs as a row in the matrices $A_{r}$ precisely $(k-n+1)$ times.

$M$ contains $(n-1)$ points. For each $a \in S-M, M \cup a$ occurs as a row precisely once in the matrix $A$. Hence $M$ occurs as a subset of precisely $(k-n+1)$ rows of $A$. Hence $M$ occurs in precisely $(k-n+1)$ rows of the matrices $A_{r}$. 
Now if $z$ is the number of different elements of $C_{q}$ and $t$ is the number of matrices $A_{r}$ in which elements of $C_{q}$ occur, then since each $A_{r}$ has $\left(C_{k, n}\right)$ rows, and each $M \in C_{q}$ occurs as a row $(k-n+1)$ times we have

$$
z(k-n+1)=t\left(\begin{array}{l}
k \\
n
\end{array}\right), \quad 0<t \leqq n .
$$

Hence

$$
z(k-n+1)=\frac{t(k !)}{n !(k-n) !}
$$

or

$$
z n=\frac{t(k !)}{(n-1) !(k-n+1) !} .
$$

But g.c.d. $(n, k ! /(n-1) !(k-n+1) !)=1$. So $n$ divides $t$. Hence $t=n$, i.e., $C_{q}=\{M\}$, and by $1.3 G$ is weakly $(n-1)$-transitive over $S$.

DEFINITIONS.

1. $a \mid b$ means $a$ divides $b$.

2. If $p, x$ are integers then $e(p, x)$ is defined to be the greatest integer $z$ such that $p^{2} \mid x$.

THEOREM 2. For every positive integer $n$ there is an integer $k \geqq 2 n$ such that g.c.d. $\left(n,\left(C_{k, n-1}\right)\right)=1$.

Proof. Let $p_{1}, p_{2}, \cdots, p_{r}$ be the distinct prime factors of $n$. For each $p_{i}$ let $a_{i}$ be an integer such that $p_{i}^{a_{i}}>n$. Let

$$
x=p_{1}^{a_{1}} \cdots p_{r}^{a_{r}} .
$$

Clearly $n \mid x$. Choose $k=x+n-1$. We first note that for every integer $h$ such that $0<h<n, e\left(p_{i}, x+h\right)=e\left(p_{i}, h\right)$. For if $p^{z} \mid(x+h)$ then since $p_{i}^{a_{i}} \mid x$ and $h<p_{i}^{a_{i}}, p_{i}^{a_{i}} \nmid(x+h)$. Hence $z<a_{i}$. But then $p_{i}^{2} \mid x$ and thus $p_{i}^{2} \mid h$. Similarly if $p_{i}^{2} \mid h$ then $z<a_{i}$ and so $p_{i}^{2} \mid(x+h)$.

Thus for $i=1,2, \cdots, r$

$$
\begin{aligned}
e\left(p_{i},\left(\begin{array}{c}
k \\
n-1
\end{array}\right)\right) & =e\left(p_{i}, \frac{(x+1)(x+2) \cdots(x+n-1)}{(n-1) !}\right) \\
& =\sum_{h=1}^{n-1} e\left(p_{i}, x+h\right)-\sum_{h=1}^{n-1} e\left(p_{i}, h\right) \\
& =\sum_{h=1}^{n-1}\left[e\left(p_{i},(x+h)\right)-e\left(p_{i}, h\right)\right] \\
& =0 .
\end{aligned}
$$


Hence g.c.d. $\left(p_{i},\left(C_{k, n-1}\right)\right)=1$. So g.c.d. $\left(n,\left(C_{k, n-1}\right)\right)=1$.

Theorem 3. Let $\tilde{S}$ be an infinite set, and $S$ an infinite subset of $\tilde{S}$. Let $G$ be weakly n-transitive over $S$. Then $G$ is weakly $(n-1)-$ transitive over $S$.

Proof. Let $X, Y$ be any two $(n-1)$-subsets of $S$. By Theorem 2 there is an integer $k \geqq 2 n$ such that $\left(n,\left(C_{k, n-1}\right)\right)=1$. Let $Z$ be a $k$-subset of $S$ such that $Z \supset X \cup Y$. Since $G$ is weakly $n$-transitive over $S$, it is weakly $n$-transitive over $Z$. But then by Theorem $1, G$ is weakly $(n-1)$-transitive over $Z$. Hence there is a $g$ in $G$ such that $g(X)=Y$.

COROllary 1. If $S$ is an infinite set and $G$ is weakly n-transitive over $S$ then $G$ is weakly $(n-1)$-transitive over $S$.

Corollary 2. If $S$ is an infinite topological space and $S$ is weakly n-homogeneous then $S$ is weakly $(n-1)$-homogeneous.

\section{REFERENCES}

1. C. E. Burgess, Some theorems on n-homogeneous continua, Proc. Amer. Math. Soc. vol. 5 (1954) pp. 136-143.

2. Certain types of homogeneous continua, Proc. Amer. Math. Soc. vol. 6 (1955) pp. 348-350.

UNIVERSITY OF WISCONSIN AND Ohio State University 